\title{
High-accuracy thrust measurements of the EMDrive and elimination of false-positive effects
}

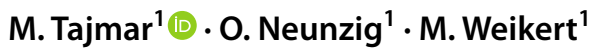

Received: 29 January 2021 / Revised: 13 July 2021 / Accepted: 15 July 2021 / Published online: 27 July 2021

(c) The Author(s) 2021

\begin{abstract}
The EMDrive is a proposed propellantless propulsion concept claiming to be many orders of magnitude more efficient than classical radiation pressure forces. It is based on microwaves, which are injected into a closed tapered cavity, producing a unidirectional thrust with values of at least $1 \mathrm{mN} / \mathrm{kW}$. This was met with high scepticism going against basic conservation laws and classical mechanics. However, several tests and theories appeared in the literature supporting this concept. Measuring a thruster with a significant thermal and mechanical load as well as high electric currents, such as those required to operate a microwave amplifier, can create numerous artefacts that produce false-positive thrust values. After many iterations, we developed an inverted counterbalanced double pendulum thrust balance, where the thruster can be mounted on a bearing below its suspension point to eliminate most thermal drift effects. In addition, the EMDrive was self-powered by a battery-pack to remove undesired interactions due to feedthroughs. We found no thrust values within a wide frequency band including several resonance frequencies and different modes. Our data limit any anomalous thrust to below the force equivalent from classical radiation for a given amount of power. This provides strong limits to all proposed theories and rules out previous test results by at least two orders of magnitude.
\end{abstract}

Keywords EMDrive - Propellantless propulsion - Thrust balance

\section{Introduction}

Propulsion systems that emit propellant are not suitable for reaching even the next star Proxima Centauri within a human lifetime. The best available technology for a crewed mission would be nuclear pulse propulsion like the one developed in project Orion, that would need 133 years for a $100 \mathrm{~m}$ diameter and 400,000 t spacecraft without considering deacceleration at the end of the journey [1]. That leaves only propellantless propulsion for much smaller robotic probes due to the low forces of radiation pressure. In principle, there are two major methods: using a sail to deflect photons or particles from the solar wind, or to use an on-board emitter

M. Tajmar

martin.tajmar@tu-dresden.de

O. Neunzig

oliver.neunzig@tu-dresden.de

M. Weikert

marcel.weikert@tu-dresden.de

1 Institute of Aerospace Engineering, Technische Universität Dresden, Marschnerstrasse 32, 01307 Dresden, Germany like a laser. The first method only works near the extrinsic power source like a terrestrial laser array or the sun whereas the second method works as long as a power source is available on the spacecraft. However, on-board photon rockets produce a very small force $F$ per input power $P$ given by $F / P=1 / c=3.3 \mathrm{nN} / \mathrm{W}$, where $c$ is the speed of light, which increases the trip time even for a very small spacecraft to thousands of years.

Already some 20 years ago, a new propellantless propulsion concept was proposed by $\mathrm{R}$. Shawyer called the EMDrive [2], which was claimed to use the difference in radiation pressure from microwaves inside a tapered cavity bouncing back and forth between the smaller and larger end to generate thrust orders of magnitude larger compared to the classical radiation pressure force. Although met with high criticism, several experimental tests and theories appeared supporting this claim. Most notably, White et al. from NASA Eagleworks published a peer-reviewed test campaign claiming a force of $1.2 \mathrm{mN} / \mathrm{kW}$ for an input power of $40-80 \mathrm{~W}$ on a torsion balance in high vacuum [3]. This is a factor of 500 higher than pure photon thrust and would therefore be of high interest if confirmed. 
In our SpaceDrive project, we are designing high-performance thrust balances with the aim to assess advanced propulsion concepts and anomalous thrust claims or theories like the EMDrive and many others [4, 5]. Previous measurements already revealed one major error source due to partially shielded cables and their interaction with the Earth's magnetic field [6]. After many iterations and improvements, we developed a setup that allows to reliably measure forces from an EMDrive similar in design to the one used by White et al. [3] with a noise level below the photon thrust threshold of $3.3 \mathrm{nN} / \mathrm{W}$, which we are using as a benchmark to compare against state-of-the-art propellantless propulsion.

This paper is structured as follows: We will first briefly review the various experimental claims and theoretical predictions, summarize the experimental difficulties and errors that we found which led to false positives, and present our consolidated final setup as well as measurements along a wide frequency spectrum including several resonance frequencies that should have produced a force. We close this paper with a conclusion and outlook.

\section{Review of EMDrive theory and experiments}

The EMDrive is a tapered cone-shaped resonant cavity fed with electromagnetic power in the microwave regime. Shawyer based his design and theory on the observation of Cullen [7], that the radiation force depends on the shape of the waveguide and therefore the group velocity. His thought then was that if microwaves are bouncing back and forth between a smaller and larger end cap, a significant net force is generated. Some designs use a dielectric disc at the smaller end to even further reduce the microwave's group velocity and increase the effect. He proposed an equation [2] for the produced force as

$F=\frac{2 \cdot P \cdot Q_{u} \cdot D_{f}}{c}$,

where $P$ is the input power to the cavity, $Q_{u}$ the unloaded quality factor (= stored energy divided by energy lost per cycle), and $D_{f}$ the design factor which depends on the used frequency and geometry of the tapered cavity. He lists typical $Q_{u}$ values as several thousands for first generation up to several ten thousands for optimized second-generation designs. The $D_{f}$ factor varies from 0.56 for a NASA-type geometry to 0.91 for further optimized designs [8]. It is more realistic to use the so-called loaded $Q$ factor, which takes port and network losses into account; however, for some of the reported measurements it is not clear which definition (loaded or unloaded) was used. From this equation, it appears that the produced thrust shall be approximately given by the standard radiation pressure force multiplied by the cavity's quality factor. This immediately raises questions on momentum and energy conservation as well as Newton's action-reaction principle. Obviously, from a textbook physics point of view, radiation bouncing back and forth will only produce heat and oscillation of the cavity, but no thrust. Shawyer claims that the conservation laws are conserved due to pre-tension in his setups, which is necessary to obtain thrust [9]. For example, this can be done with a counterweight on a weight balance or with two EMDrives pointing in opposite directions. Alternatively, no pre-tension is necessary if the EMDrive is operated with amplitude modulation or essentially in pulsed mode with frequencies at 50 and $300 \mathrm{~Hz}$, which showed good results.

Some theories have appeared that support Shawyer's claim. For example, McCulloch proposed a theory [10] based on inertial modification due to Unruh radiation giving a similar force prediction as Eq. (1) with a design factor close to unity. However, in his definition, $Q$ is not the quality factor but the number of reflections within the cavity (which is typically much less). He later refined his model taking the dielectric into account [11]. White et al. [3] proposed that the EMDrive directly interacts with the background zeropoint-fluctuations of the vacuum to create propellantless thrust. Smolyaninov [12] suggested that the electromagnetic fields in the cavity may create Axion-like particles as an optical analog of the Schwinger effect that could be responsible for the observed forces. Last but not least, Grahn et al. [13] assume that photons may in fact leave the closed cavity in the form of phase-opposing and thus E-field-free photon pairs.

Of course, all these theories are highly controversial [14] as any observation of forces higher than photon thrust is outside mainstream physics. They rely on the fact that the claimed thrusts are real, and therefore, we will concentrate on the best experimental evidence as the baseline for our investigation.

Shawyer published three detailed test reports on his webpage, which used electronic balances at ambient pressure as the force transducer. The first one conducted several experiments with a thruster operating at a power of $850 \mathrm{~W}$ and a resonance frequency of about $2.45 \mathrm{GHz}$ with a dielectric at the smaller end [15]. The microwaves were generated from a magnetron powered by a half wave rectified high-voltage power supply at $50 \mathrm{~Hz}$ resulting in pulsed operation. Three different balance configurations were used including a beam balance with counterweight, an electronic balance at the bottom with the EMDrive on top and a spring that connected it to a fixed top structure, as well as a horizontal variant with a pivot that converted horizontal movement into a force again pushing on an electronic balance. $Q$ values were ranging from 2500 to 5900 and the forces measured were in the range of $10 \mathrm{mN}$ in line with the prediction of Eq. (1). Different 
orientations seemed to be consistent with a real thrust, such that the thruster pointing upwards and downwards produced thrusts with changing signs, and pointing perpendicular with respect to the force measurement direction produced zero thrust. Moreover, various spurious effects were analyzed and dismissed such as buoyancy, any influence from the Earth's magnetic field, or the cooling fans from the magnetron.

The second test report [16] details a more efficient thruster without dielectric powered again by a magnetron and without pulsed operation. With a $Q$ factor of 19,521, a thrust of $82 \mathrm{mN}$ was measured for an input power of $744 \mathrm{~W}$. This was verified again in a vertical and horizontal measurement with different thruster orientations.

In the third test [17], a so-called C-Band flight thruster without dielectric operated at $3.85 \mathrm{GHz}$, and with approximately $300 \mathrm{~W}$ as well as a $Q$ factor of 55,172 produced a force of around $100 \mathrm{mN}$. A traveling wave tube amplifier (TWTA) was used instead of the magnetron without any mentioning of pulsed outputs, so we can also assume continuous operation. In addition, a contactless connection between amplifier and cavity was used without further details. In the test report, no information was given on force measurements for different thruster orientations or discussions on possible spurious effects, and only a vertical measurement was described with the thruster hanging down from a spring and touching the balance on the bottom. Another test is only briefly described, where a $100 \mathrm{~kg}$ test rig consisting of the EMDrive and all associated electronics is mounted on an air bearing with flexible cables providing power [18]. It is claimed that $334 \mathrm{~W}$ produced a force of $96 \mathrm{mN}$ and a corresponding rotary motion on the air bearing, but unfortunately, no test data or analysis with different thruster orientations was made available.

Yang et al. [19] initially reported very high thrust measurements for an EMDrive on a force-feedback thrust stand powered by a magnetron at $2.45 \mathrm{GHz}$ with a power ranging from 80 to $2500 \mathrm{~W}$ and corresponding forces of $70-720 \mathrm{mN}$. However, later measurements with a different wire torsion pendulum did not show any thrust higher than their measurement uncertainty of $0.7 \mathrm{mN}$ at a power of $230 \mathrm{~W}$ [20]. Fetta [21] reported thrust generated from a pancake-shaped cavity at $937 \mathrm{MHz}$ with very high $Q$ values of $10^{7}$ just below liquid helium temperatures of around $10 \mathrm{mN}$ for an input power of $10.5 \mathrm{~W}$. However, his setup and measurement is poorly described. Brady et al. [22] further tested Fetta's design on a torsion balance obtaining $22.5 \mu \mathrm{N}$ with an input power of $28 \mathrm{~W}$ at room temperature using a standard solid-state amplifier and a PTFE dielectric at one end.

White et al. [3] performed an extensive test campaign in high vacuum on a torsion balance test stand with an EMDrive using a tapered cavity with a dielectric at the smaller end, a frequency of $1937 \mathrm{MHz}$, power levels ranging from $40-80 \mathrm{~W}$, a quality factor of 7123 and thrusts ranging from 30 to $130 \mu \mathrm{N}$, which is around an order of magnitude below Eq. (1). Considering that an unloaded $Q$ factor is usually higher than the loaded one (including losses from all electronic components), the fit should be even better. Tests were done in forward, reverse, and perpendicular (=null-thrust) orientation with corresponding thrust measurements consistent with a real thrust. All electronic components including a solid-state amplifier were mounted on the balance powered through liquid metal contacts. Resonance frequency tracking was implemented using a digital closedloop control. The observed thrust slopes were superimposed on thermal drifts of similar magnitude. At the end, the paper lists a number of possible error sources which were analyzed and dismissed including air currents, radiofrequency (RF) and electromagnetic interactions, thermal issues, and outgassing. McDonald [23] published a test setup using also White's cavity geometry and a frictionless finger-joint/ RF coupling technique to further reduce electromagnetic interactions. Due to the depth of their assessments, we will concentrate our own experimental assessment on the White cavity design.

Sokoloff et al. [24] recently published a test of two identical EMDrives in opposite directions on top of an electronic balance that can be operated individually to check for corresponding upwards and downwards forces without modifying the setup. Due to the high weight and stiff coaxial cable from the amplifier, no force was measured for a thruster operating with $2.45 \mathrm{GHz}$, a $Q$ factor of 3550 , and a power of $140 \mathrm{~W}$ within their measurement resolution of $0.5 \mathrm{mN}$. Shortly afterward, an update was published by Peyre et al. [25] with a contactless microwave connection and an increased $Q$ factor of up to 18,500 , where no force was measured down to $0.1 \mathrm{mN}$ for a power of $150 \mathrm{~W}$ fed into the cavity. Recently, this test was repeated with a cavity including a dielectric insert with a $Q$ factor of 7,000 at a power of $160 \mathrm{~W}$ with the same mode (TM212) as White et al. [3], and again, no force was measured [26].

Our own tests started with an EMDrive using a $2.45 \mathrm{GHz}$ microwave oven magnetron at $700 \mathrm{~W}$ with a $Q$ factor of 50 on a beam balance at ambient pressure [27], which showed a difference between upwards, downwards, and horizontal orientation but large thermal drifts in the order of hundreds of $\mu \mathrm{N}$. The same thruster was then mounted on a torsion balance in high vacuum and did not show thrusts higher than our error bar of $\pm 20 \mu \mathrm{N}$.

Next, we replicated the geometry used by White et al. [3] including the dielectric end plate and significantly upgraded our balance and electronics [6]. Using a much larger vacuum chamber, a higher sensitivity torsion balance, and a solidstate amplifier, we obtained similar values as others such as a $Q$ factor of 2000 (loaded value based on our definition used in this paper) and a thrust that changed sign depending on its orientation in the range of 3-6 $\mu \mathrm{N}$ for an input power of $2 \mathrm{~W}$. 
This is a bit less compared to what we would expect from Eq. (1); however, the $Q$ factor might have been too high. The force-to-power ratio of around $1 \mu \mathrm{N} / \mathrm{W}$ or $1 \mathrm{mN} / \mathrm{kW}$ was similar to the one reported by White et al. [3]. However, our test setup included an optional $40 \mathrm{~dB}$ attenuator that basically eliminated all microwaves going into the cavity while leaving the rest of the setup identical to the previous measurement. Tests with the $40 \mathrm{~dB}$ attenuator showed similar thrust values compared to without it, indicating that these forces must be due to something else than the microwaves in the cavity. Our best estimate was an interaction of just a few centimeters of unshielded cables with the Earth's magnetic field that produced similar forces. Further work concentrated on trying out liquid-metal RF feedthroughs, which placed the amplifier with its high DC currents and thermal load outside the chamber [5], as well as the development of a continuously rotating thrust stand using superconducting levitation [28] to investigate the reality of the observed forces.

Last but not least, Taylor predicted that an EMDrive operating at optical frequencies instead of microwaves might be more compact and efficient [29]. This was experimentally investigated by ourselves in a separate paper [30]. The continuous development of the various setups provided us with a good understanding of different experimental artefacts and led to the development of a reliable setup that eliminated spurious interactions and drastically boosted our sensitivity to below the photon pressure force, which is our design benchmark for propellantless propulsion. Given the power levels involved, this required sub- $\mu \mathrm{N}$ resolution for hundreds of Watts to just a few $\mathrm{nN}$ for a few Watts of input power.
A summary of all published measurements and a comparison to the data obtained in this paper is shown in Table 1.

\section{Experimental difficulties}

So far, we have seen two different measurement principles: an electronic balance using weight changes or a torsion balance that uses deflection and a spring constant to calculate the force generated by the EMDrive. There are many thruster-induced and environmental interactions that can easily generate false-positive thrust signatures similar to the ones reported above. Here is a list of the most important ones that we observed:

1. Buoyancy, atmospheric interactions, and outgassing: Apart from the fact that an ambient atmosphere causes noise and therefore may prohibit any balance to reach the sensitivity required, buoyancy is a significant factor for the forces involved. Injecting tens to hundreds of Watts into a cavity causes the thruster and its electronics to heat up significantly. If we consider a typical cavity volume of $15 \times 15 \times 15 \mathrm{~cm}^{3}$, a temperature change of only one degree Celsius creates a lifting force of $150 \mu \mathrm{N}$ ! This severely affects all weight measurements in ambient conditions. In vacuum, gases can cause problems too. For example, some residual gas or other components that can easily evaporate may be trapped. Heating up the thruster can cause outgassing in one direction that can create forces which mimic a real

Table 1 Summary of past experiments and comparison to this work as well as the photon thrust limit

\begin{tabular}{|c|c|c|c|c|c|c|c|}
\hline Reference & Dielectric & Power source & Frequency $[\mathrm{MHz}]$ & Loaded $Q$ factor (Mode) & Cavity power [W] & Thrust $[\mu \mathrm{N}]$ & $T / P$ ratio $[\mu \mathrm{N} / \mathrm{W}]$ \\
\hline Shawyer [15] & Yes & Magnetron & 2450 & $2500-5900^{\mathrm{a}}$ & 850 & 10,000 & 12 \\
\hline Shawyer [16] & No & Magnetron & 2450 & $19,521^{\mathrm{a}}$ & 744 & 82,000 & 110 \\
\hline Shawyer [17] & No & TWTA & 3850 & $55,172^{\mathrm{a}}$ & 300 & $\approx 100,000$ & 326 \\
\hline Yang [20] & No & Magnetron & 2450 & N/A & 230 & $<700$ & $<3$ \\
\hline Brady [22] & Yes & Solid State & 937 & N/A & 28 & 22.5 & 0.8 \\
\hline White [3] & Yes & Solid State & 1937 & 7,123 (TM212) & $40-80$ & $30-130$ & $\approx 1.2$ \\
\hline Sokoloff [24] & No & Solid State & 2441 & 3550 & 140 & $<500$ & $<3.6$ \\
\hline Peyre [25] & No & Solid State & 2455 & 18,500 & 150 & $<100$ & $<0.7$ \\
\hline Peyre [26] & Yes & Solid State & 2464 & 7000 (TM212) & 160 & $<100$ & $<0.6$ \\
\hline Tajmar [27] & No & Magnetron & 2450 & 50 & 700 & $<20$ & $<0.03$ \\
\hline Tajmar [6] & No & Solid State & 1865 & 2000 & 2 & $<1$ & $<0.5$ \\
\hline \multirow[t]{4}{*}{ This work } & Yes & Solid State & 1857 & 741 (Hybrid) & 6 & $0.003 \pm 0.02$ & $<0.003$ \\
\hline & & & 1914 & 477 (TE312) & 6.5 & $0.007 \pm 0.01$ & $<0.002$ \\
\hline & & & 1,943 & 430 (TM212) & 3 & $-0.009 \pm 0.01$ & $<0.003$ \\
\hline & & & 1984 & 330 (Hybrid) & 7 & $0.004 \pm 0.02$ & $<0.003$ \\
\hline Photon thrust & & & & & & & 0.0033 \\
\hline
\end{tabular}

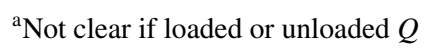


EMDrive force, which even changes signs correctly with different thruster orientations.

2. Magnetic interactions: The amplifiers on the EMDrive need high currents of at least several Amperes. Cables going to the electronics can be twisted to reduce external magnetic fields. However, no shielding is perfect and there are always little paths without twisting that add up to non-negligible fields. This can then interact with the Earth's magnetic field or with permanent magnets in close vicinity. Examples are magnets used for eddy-current damping of the balance or magnets in vacuum gauges or turbo pumps, which are mounted on the chamber walls. Reorienting the thruster can also change the direction of the force if those external magnets are mounted off-axis. It is therefore very important to map the magnetic environment close to the balance to remove such obstacles. In White et al.'s setup [3], the damping magnet was mounted on the external supporting structure and not on the balance arm, which can very well cause orientation-dependent magnetic interaction forces of similar magnitude compared to their claimed thrusts. Another non-intuitive magnetic interaction is that high-frequency fields can induce currents in voicecoils, which are frequently used to calibrate balances. A DC power supply connected to the coil can rectify the signal causing a real force [31].

3. Feedthrough interactions: So far, three different feedthroughs were used to provide power to the thruster or read back monitoring signals: flexible cables, liquid-metal pin contacts, and contactless RF joints which require very precise alignments. The first method is obviously the worst choice as it introduces an additional spring constant that can create large forces. They can have a strong temperature dependence, which in turn can lead to false positives rendering sub- $\mu \mathrm{N}$ measurements impossible. Liquid-metal contacts seem like a good choice on first sight and were used on many of the previous tests as the liquid removes the cable's stiffness. However, we found out that surface tension forces between the pin and the liquid exist that depend on the current passing through. This can lead to several $\mu \mathrm{N}$ for currents required to power the amplifier. In addition, careful positioning of the feedthrough is required to limit their interaction with the overall thrust measurement.

4. Thermal interactions: This is one of the most important interactions, which may explain a majority of the claimed thrust measurements. Heating up of the cavity or the electronics during operation causes thermal expansion which in turn shifts the center of gravity. Torsion balances are very sensitive to such shifts causing motion of the balance to its new zero position. This can easily be misinterpreted as a real thrust with the correct signature as changing thruster orientations can lead to a change in the new zero position too. Also, weight balances show changes if the center of gravity moves above their suspension point, because it creates a torque. That is why most electronic balances feature a hook to weigh samples below it connected to a single point in the middle to remove this artefact. However, all tests using this method so far like the ones from Shawyer [15-17], positioned the EMDrive on top of the balance. Tests performed by us with samples that can heat up show a significant difference if weighted above or below on a hook due to this effect [32]. Specifically, the EMDrive with its large volume usually made from copper or steel acting as a heat sink is very sensitive to this. In addition, heating up of the cavity is linked to the thruster's resonance frequencies, which can produce very convincing thrust signatures. Not only the thruster is susceptible to thermal drifts, but also the balance itself consisting of its arms and spring can be influenced by varying temperatures. A change in the spring constant directly translates into deflections that are interpreted as forces. This can be even amplified by mechanical fixation of some parts of the thruster to the balance arm, which results in large false-positive forces. In addition to real thrust-mimicking plateaus, thermal drifts are always present and are usually superimposed on any measurement. McDonald tried to remove them by heating up the whole balancethruster assembly to a constant temperature [23]. Software tools are necessary to automatically detect such drifts and systematically remove them to achieve high resolution.

In addition to taking care of such thruster-environment interactions, it is very important to have a reliable null-measurement, e.g., by having the thruster pointing in a direction perpendicular to the measurement axis. Many iterations in the setup both on the thruster and the balance are necessary to determine if a signal is real or an artefact.

\section{Experimental setup}

Here, we describe our final configuration, which addressed all the artefacts that we observed to achieve a reliable and high-sensitivity measurement to assess, if the EMDrive produces any force higher than a pure photon thruster. In short, our key components are as follows:

1. A new type of thrust balance was designed that was much less sensitive to center of gravity shifts.

2. The thruster was mounted on a bearing below its suspension point to further reduce thermal induced expansion shifts or mechanical stress on the balance and its pivots. 
3. Magnetic components were removed or relocated as much as possible like the eddy-current damping magnet or vacuum chamber components until the magnetic environment did not cause any measurable effect.

4. The EMDrive was mounted as a "black box" on the thrust balance. Using a battery-pack to power the amplifier and required electronics, we eliminated all feedthrough (high currents, RF) issues. Only data monitoring and commanding signal connections used a liquid-metal pin contact but at such low power that the influence was below our resolution. This complete assembly should also represent a real application like operating a thruster on a satellite as close as possible.

A detailed description is summarized as follows.

\subsection{Inverted counterbalanced double pendulum}

Considering the weight of the whole EMDrive assembly of $9 \mathrm{~kg}$, a weight balance is not an option to reach radiation pressure resolution. On the other hand, a classical torsion balance as used by White, McDonald, and ourselves, which consists of a single arm and a torsion spring in the middle, turned out far too sensitive to center of gravity shifts due to thermal expansion. We therefore developed an inverted counterbalanced double pendulum [33], as illustrated in Fig. 1. Instead of a single arm, it consists of two platforms, which drastically reduces the influence of the actual position of the center of gravity. The top square hosts the thruster and the counterweights are located at the bottom. A tripod connects the two platforms with a total of nine frictionless flexural pivots. The deflection is measured using an attocube IDS3010 laser interferometer with a $10 \mathrm{~Hz}$ low-pass filter. Calibration of the balance is performed using a voice-coil, which is commanded using a Keithley 2450 precision current source. This converts the observed deflection into force. The voice-coil itself was calibrated and compared against its datasheet force constant using a Sartorius AX224 balance. The resulting calibration constant was highly linear within our measurement range, as shown in Fig. 2. To remove any permanent magnet in the vicinity of the thruster, we decided to implement damping as a closed-loop software solution
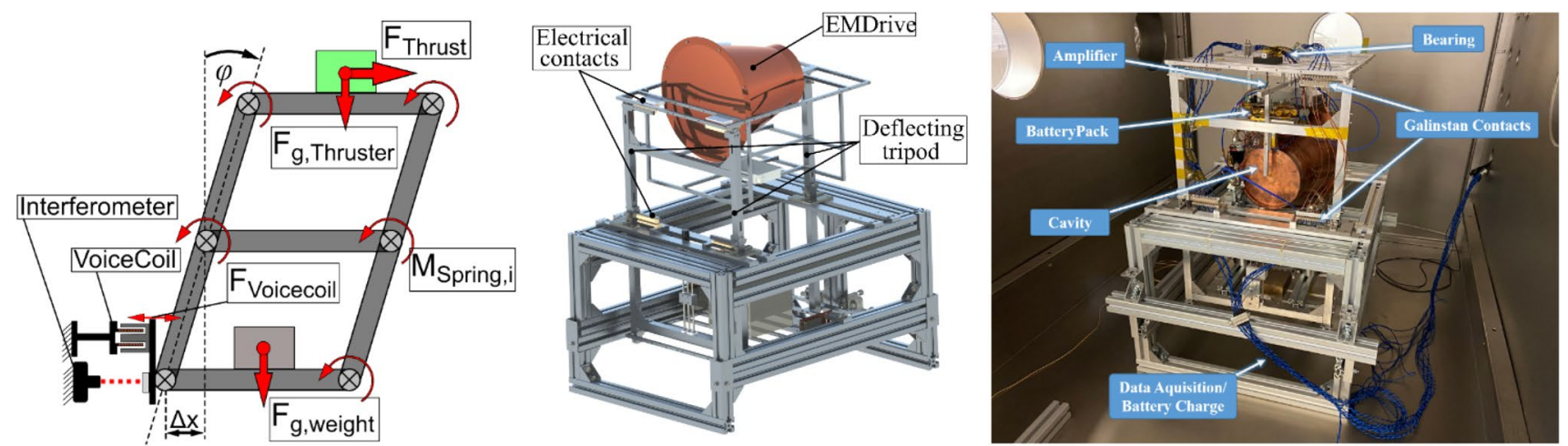

Fig. 1 Schematic illustration of inverted counter balanced double pendulum and position in vacuum chamber
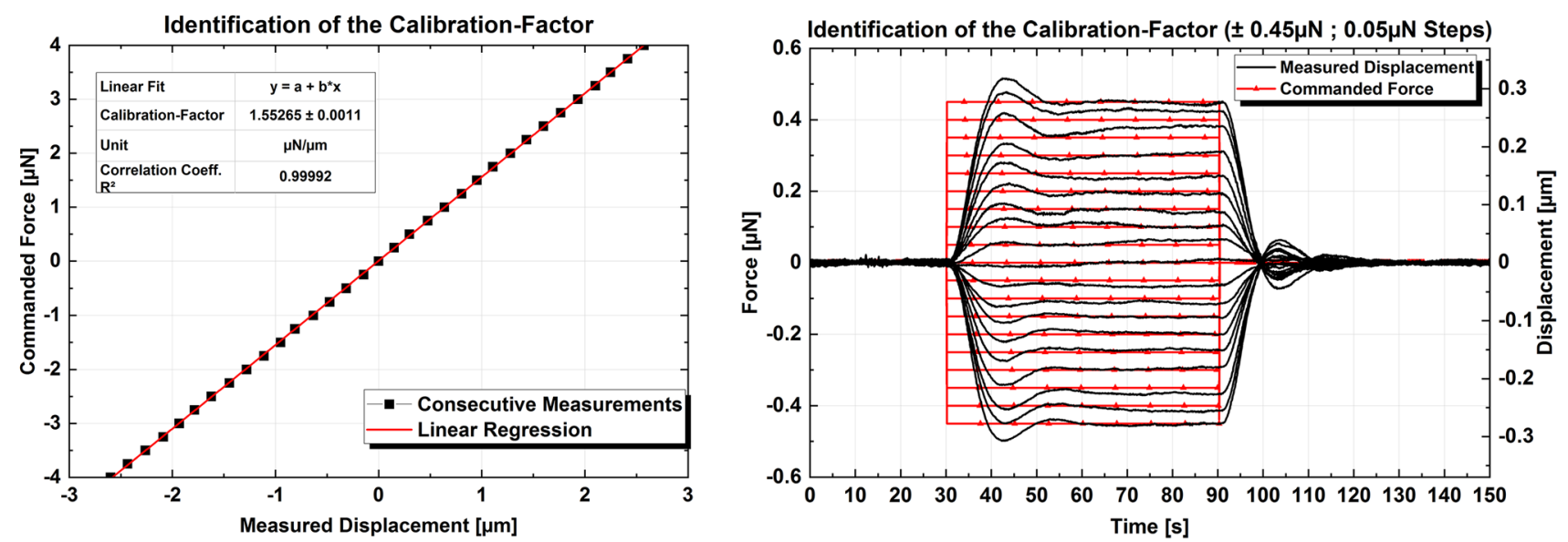

Fig. 2 Balance calibration 


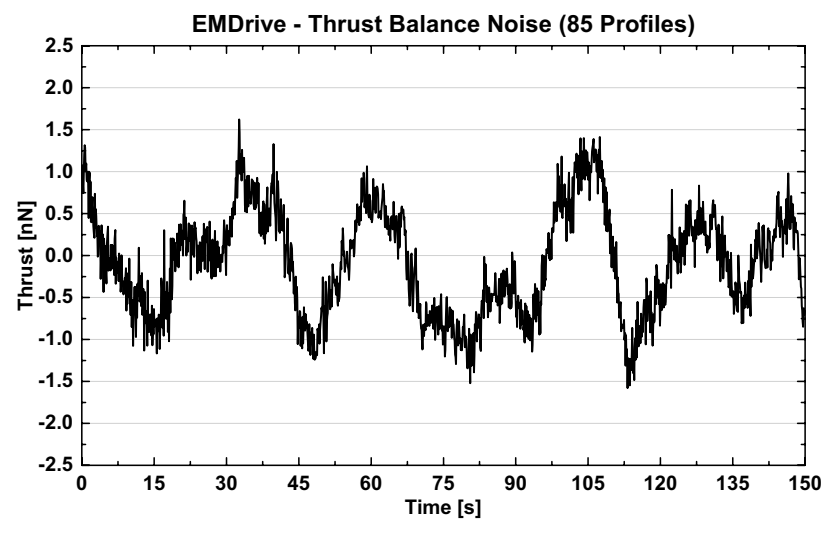

Fig. 3 Thrust noise example

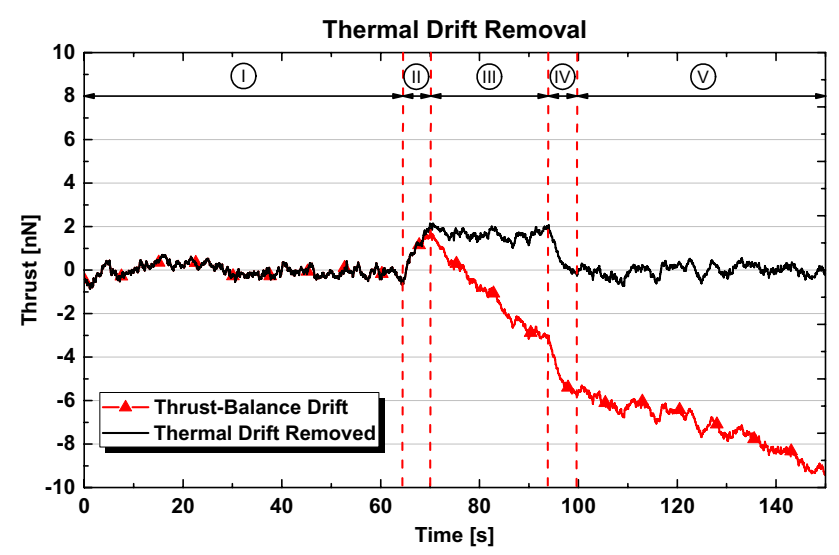

Fig. 4 Example of thermal drift compensation and sector classification (I, V off, II ramp up, III on, IV ramp down)

using the voice-coil instead of the usual eddy-current permanent-magnet-copper plate assembly. Proper counterweights and the selection of a critical damping constant resulted in a reaction time of about $15 \mathrm{~s}$ and an exceptional low noise, as shown in Fig. 3, while the whole setup was mounted but non-operational. Using data averaging with many measurements, sub-nN resolution could be obtained as required. This is the lowest noise level by many orders of magnitude of thrust balances with a weight capacity of up to $10 \mathrm{~kg}$ that we have found in the literature.

Each measurement was subdivided into different sectors of fixed durations with an initial off-period, then ramping up to the desired power followed again by ramping down to zero and maintaining a second off-period. This allows to systematically analyze drifts from the laser interferometer during the off-periods as well as typical linear thermal drifts during the on-periods. A LabView program was developed to automatically detect and compensate such drifts, as illustrated in Fig. 4. A script-based program controlled both balance and thruster and performed a complete set of calibration before and after each measurement campaign as well as executing any measurements with a pre-defined number of profiles to gain statistical significance and to improve noise.

Finally, the balance with approximate dimensions of $550 \times 740 \times 700 \mathrm{~mm}^{3}$ was put on passive dampers into a large stainless-steel vacuum chamber with dimensions $1.2 \times 1.5 \times 2.5 \mathrm{~m}^{3}$, which provided sufficient distance to the walls as well as pumps and gauges to eliminate electromagnetic interactions. An Edwards's oil-free scroll pump was used to reach $10^{-2}$ mbar, which was sufficient to avoid buoyancy and allowed quick turn-around times to iterate or change setups on a daily basis. The balance featured up to 20 liquid-metal pin contacts that were optimized for vacuum as well as minimum forces due to currents passing through them. All cables were twisted in pairs to reduce magnetic fields as much as possible. An FLIR thermal camera was mounted inside the chamber to observe the whole assembly and to identify hot spots, which would cause thermal drifts.

Most important was mounting of the EMDrive to the balance platform. To eliminate the sensitivity to thermal drifts and center of gravity shifts, the assembly was mounted hanging down from a bearing, which consisted of a simple bolt in the middle of the upper platform with its rotation axis perpendicular to the thrust direction. This is similar to the hook configuration on weight balances and proofed to be very effective. To illustrate the influence of mounting, we performed the same measurement in three different configurations, as shown in Fig. 5: in position A, the EMDrive was mounted on a cage-like mounting structure that made its center line align with the top platform; in position B, the cage was removed and the EMDrive was put standing on dampers on the top platform, and finally, in position C, where the thruster package was mounted on the bolt bearing hanging down from the middle point of the thruster platform.

The corresponding measurements for identical operating conditions in frequency and power are shown in Fig. 6A, where the amplifier current indicates when the thruster was on. Position A produced a large force consisting of a plateau and a drift similar to the ones reported in White et al. [3] with $1.5 \mu \mathrm{N}$ for an input power of $10 \mathrm{~W}$. This could be amplified by mechanically fixing the cavity to the cage rods with screws raising the thrust level to $20 \mu \mathrm{N}$, as shown in Fig. 6B. That value is similar to what we expected from White et al. [3] with a claimed thrust-to-power ratio of about $1 \mu \mathrm{N} / \mathrm{W}$. However, this was not a real thrust but originated from mechanical stress due to the thermally expanding cavity, which acted on the balance's pivots. In position B, mechanical stress was reduced by eliminating the cage; however, small center of gravity shifts due to thermal expansion were still present and a thrust of around $200 \mathrm{nN}$ was present. Only after choosing position $\mathrm{C}$, the thrust artefacts disappeared and only noise was left. 


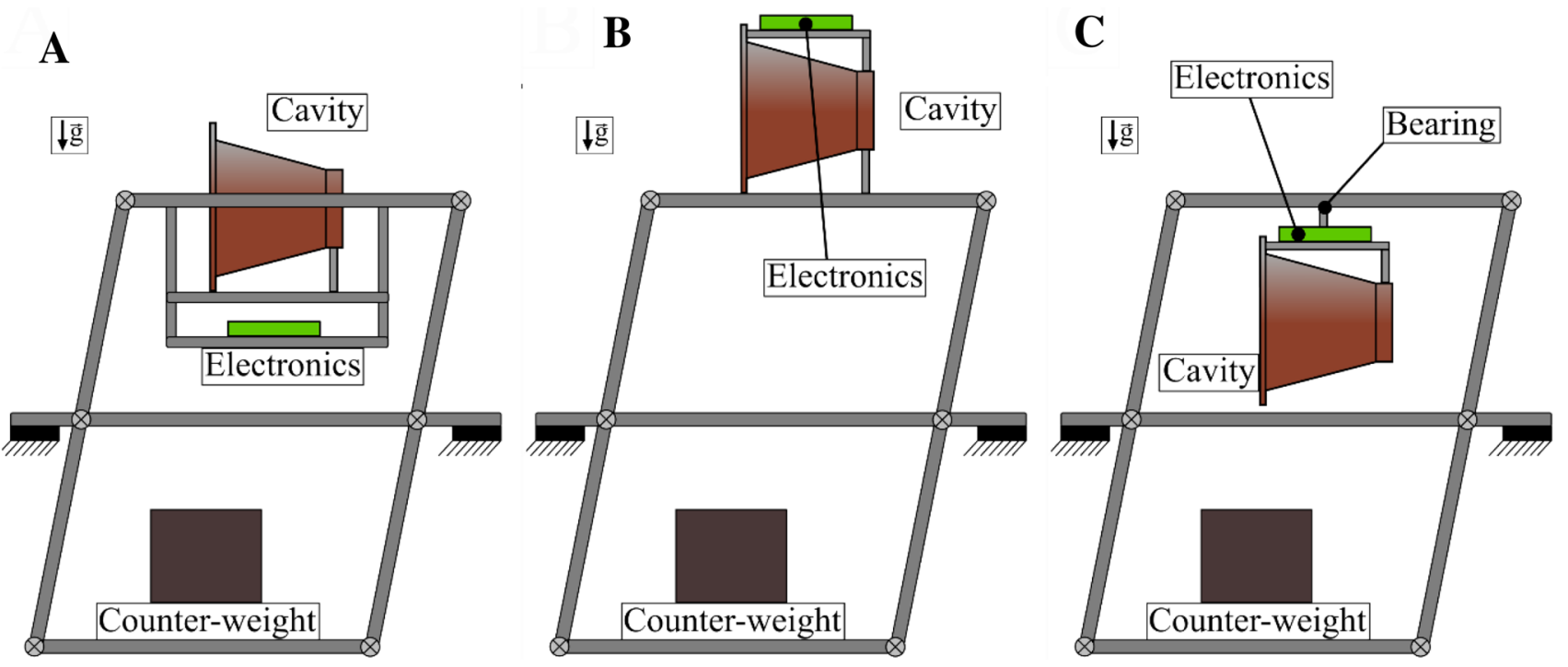

Fig. 5 Different mounting configuration of EMDrive on thrust balance (A on cage, $\mathbf{B}$ on top of platform, $\mathbf{C}$ hanging from bolt bearing)

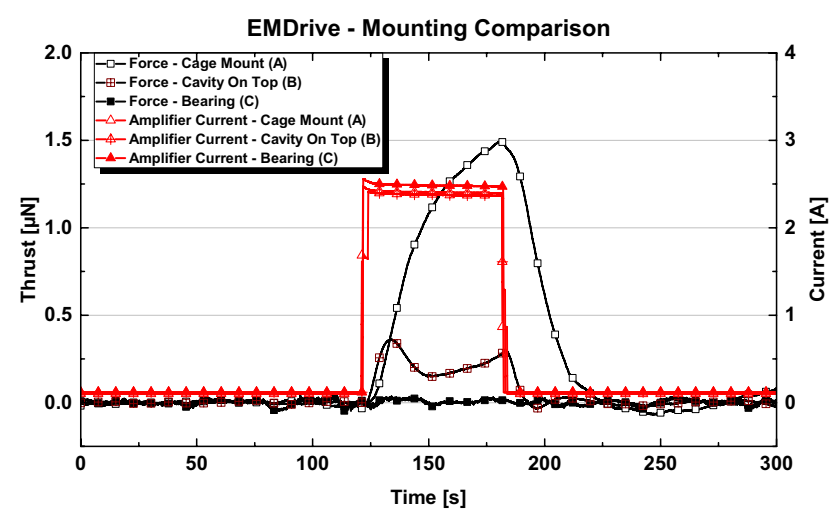

A Comparison between Mounting Options A-C

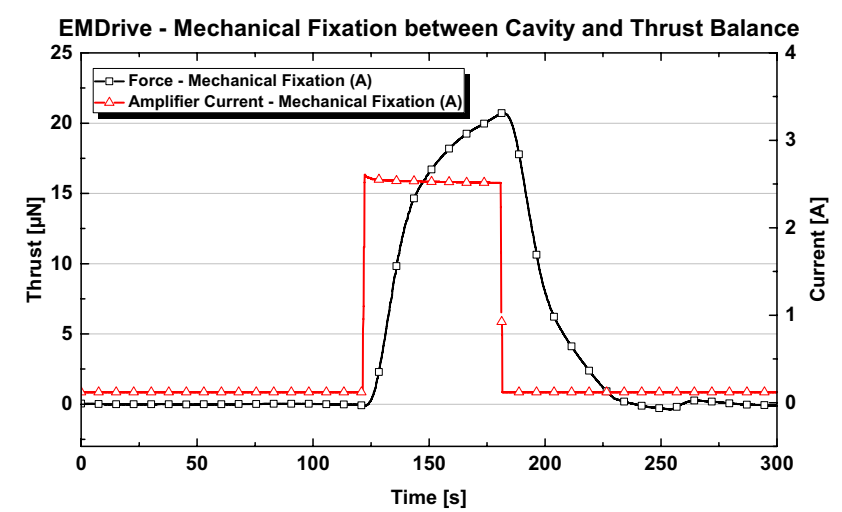

B Option A with Mechanical Fixation of Cavity on Cage

Fig. 6 False-positive thrust signals

\subsection{EMDrive and electrical setup}

Our cavity is based on the same geometry as the one used by White et al. [3] to compare with peer-reviewed results. It consists of a tapered cone, which was mechanically pressed, with a cylindrical flange and a collar made out of 2 mm-thick copper, as shown in Fig. 7. The large flat end surface can be screwed onto the collar and the smaller end surface can slide into the cylindrical flange with a tight fit, which allows fine tuning. Two $20 \mathrm{~mm}$ - and one $14 \mathrm{~mm}$ thick HDPE discs with a diameter of $155 \mathrm{~mm}$ are glued together and fixed to the end surface using Scotch-Weld 2216 adhesive. A loop antenna made of $1 \mathrm{~mm}$-thick copper wire with a diameter of approximately $13 \mathrm{~mm}$ is used for the power feed. The antenna is screwed into the cavity

using an N-type connector. For this purpose, a position was chosen, which, according to COMSOL simulations, lies within the range of high magnetic field strengths of various modes. The antenna can be aligned horizontally or vertically to improve magnetic field excitation in the cavity.

To eliminate feedthrough problems due to high currents or RF signals, we decided to operate the EMDrive with a battery-pack that provided enough power for all components during a whole night, which we preferred for measurements due to low seismic noise. Only monitor and command signals were still routed through the liquid-metal pin contacts during operation. The following electronic components completed our circuit, as shown in Fig. 8: 


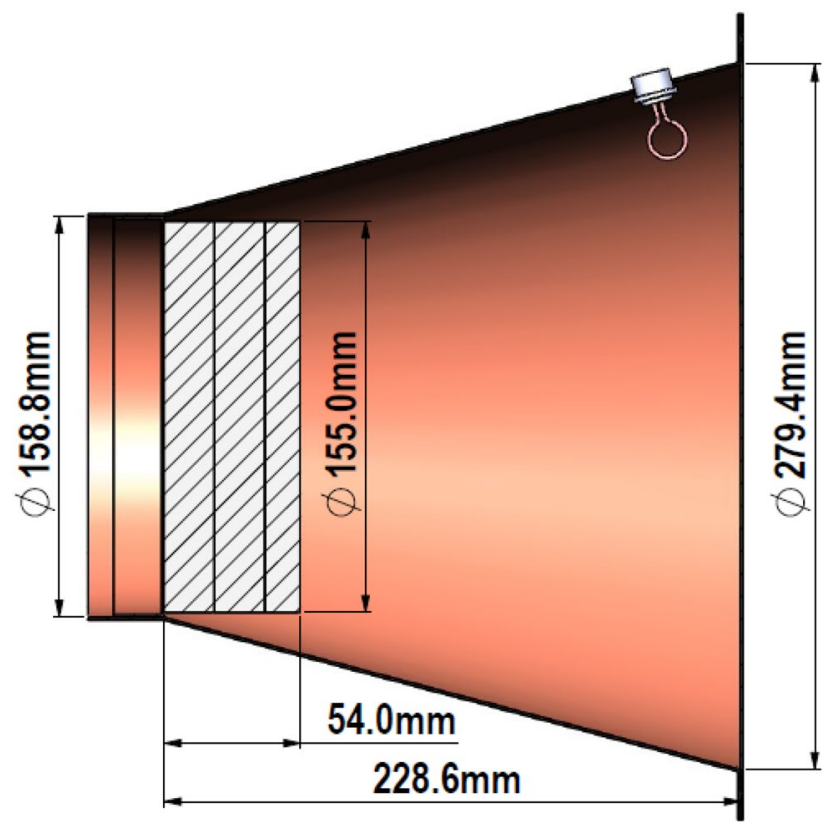

Fig. 7 EMDrive cavity dimensions with two HDPE dielectric discs (Shaded Region)

- Battery-pack with six 18,650 Lithium-Ion cells and balancing electronics as well as DC-DC converters for all other components. This was re-charged before any measurement campaign.
- Mini-Circuits ZX95-2041-S + voltage-controlled frequency generator.

- Mini-Circuits ZX73-2500-S + voltage-controlled attenuator to control the output power.

- EMPower SKU 1164 RF solid-state amplifier with a maximum output power of $50 \mathrm{~W}$ and an amplification of $47 \mathrm{~dB}$ according to our measurements.

- MECA CN-1.950 circulator that prohibits damage to the amplifier by re-directing any reflected power to a separate output, which was measured by a Mini-Circuits BW-N30W20 + fixed attenuator and a Mini-Circuit XZ47-40LN-S + power meter. This is similar to the setup used by Shawyer [17].

- Maury Microwave 1878B three-stub tuner for impedance matching directly next to the antenna.

This enabled us to operate the EMDrive at a wide frequency range and at power levels comparable to White et al. [3]. However, we chose a cavity input power of around $10 \mathrm{~W}$ compared to $40-80 \mathrm{~W}$ by White et al. due to our battery and to reduce thermal load. Nevertheless, the power values in our setup are also more reliable as we only used a circulator and one power meter instead of a directional coupler like White et al., which we found to produce values that can be significantly off-value due to reflections from the cavity. The amplifier had a more or less constant power consumption when turned on consuming $2.5 \mathrm{~A}$ at a battery voltage of

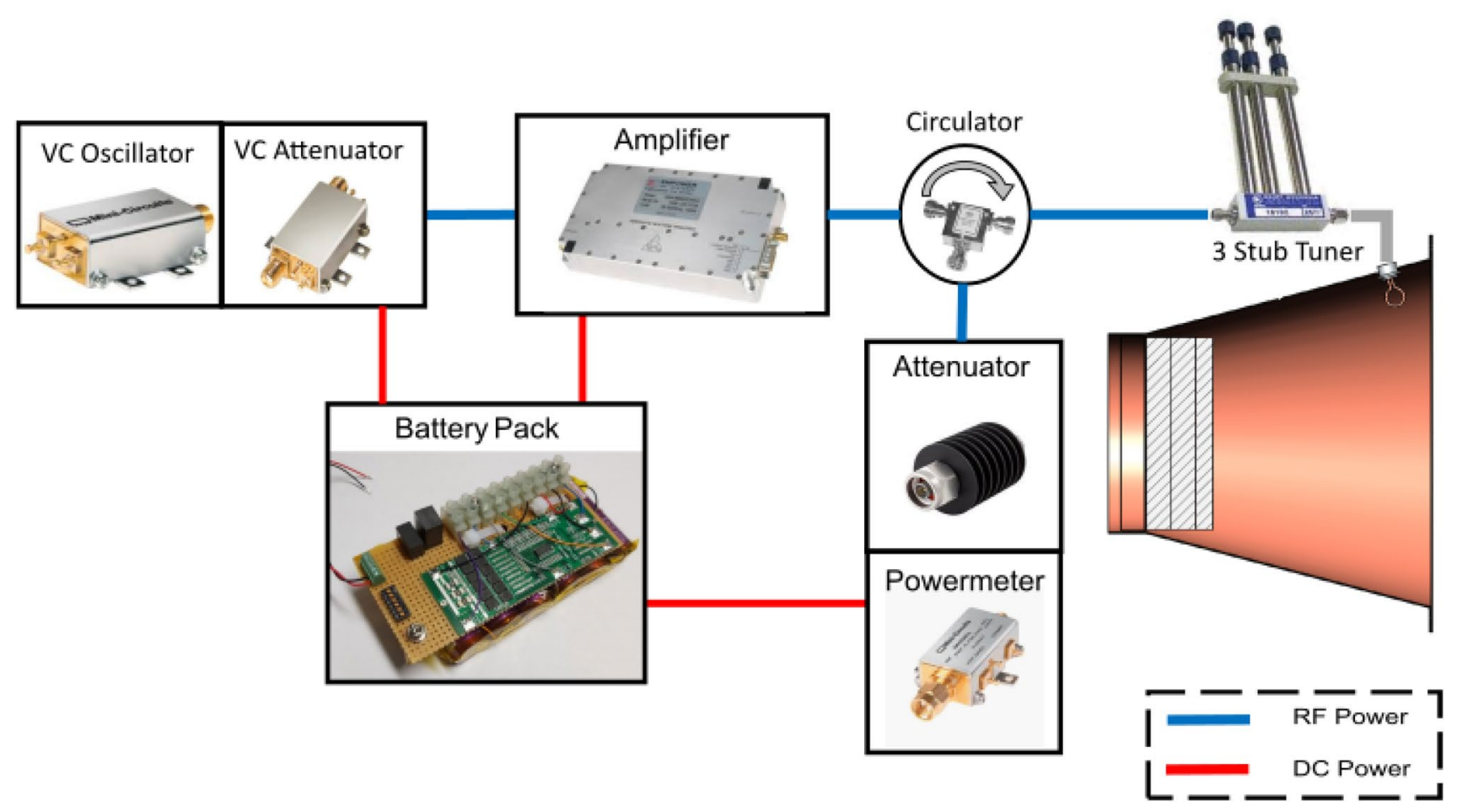

Fig. 8 Electrical setup 
24.6 V. This is more than $60 \mathrm{~W}$ and comparable to White's input power. The overall power consumption was even $10 \%$ higher taking into account all other electronics in our setup. All components are frequency- and temperature-dependent. Therefore, their characteristics were evaluated before use and calibrated.

To provide maximum power transfer from the power generating system into the cavity, it has to be matched to the $50 \Omega$ wave-impedance coaxial components. Typically, this matching occurs at a resonant frequency. Further fine-tuning by applying additional capacitive and inductive parts to the wave impedance can be realized with a three-stub tuner. For fast and precise tuning, a single-port Vector Network Analyzer (VNA) Anritsu MS46121A was used. The EMDrive was matched to a resonant frequency of $1,914 \mathrm{MHz}$, which was close to the $1,937 \mathrm{MHz}$ used by White et al. [3]. We scanned from around $1,850 \mathrm{MHz}$ to above $2,000 \mathrm{MHz}$ and could identify also other resonance frequencies, as shown in Fig. 9. COMSOL electromagnetic field simulations were done to identify the excited

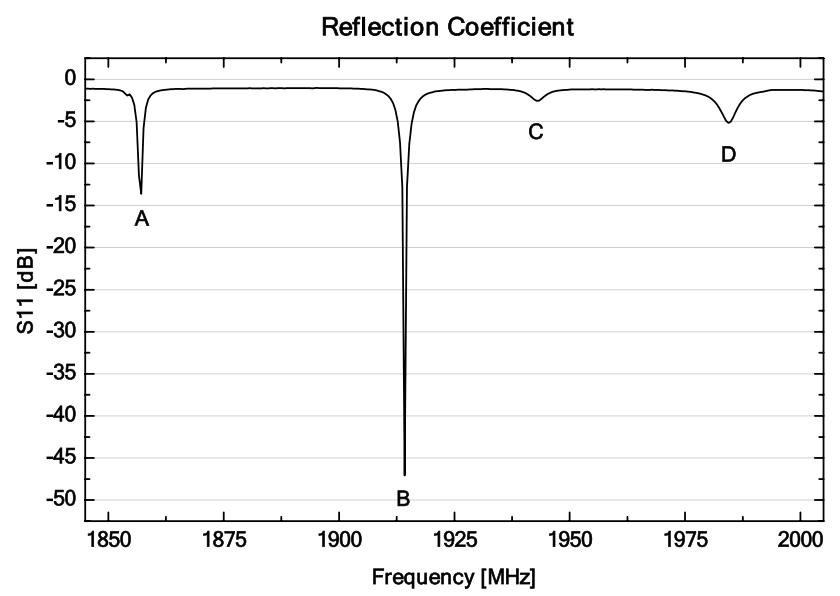

Fig. 9 Reflection coefficient measurement, A-D Correspond to Modes in Fig. 10. (Loaded $Q_{A}=741, Q_{B}=477, Q_{C}=430, Q_{D}=330$ ) using $Q=f / \Delta \mathrm{f}_{\text {half-power }}[34]$ modes, as shown in Fig. 10 (resonance peaks A-D with corresponding mode shapes and $Q$ factors). The loaded $Q$ factor is usually determined by using $Q=f / \Delta f_{-3 \mathrm{~dB}}$ at the resonance peak; however, as our data also include peaks with less than $3 \mathrm{~dB}$, a more general procedure was used as described in the literature [34], where $Q$ is determined by the ratio of the $\mathrm{S} 11$ values at resonance and the frequency bandwidth at half power, $Q=f / \Delta f_{\text {half-power }}$.

Our peaks also include the TM212 mode (used by White et al.) at a resonance of $1943 \mathrm{MHz}$ with a $Q$ factor of 430. This is around an order of magnitude below White et al.'s calculated 7123 , probably due to deviations in the surface roughness, antenna alignment, and tuning for a wider bandwidth to test multiple modes. Still, we consider this a good match and, as we will show later, our thrust sensitivity is as low as a $Q$ factor of only one. At our tuned resonance frequency of $1914 \mathrm{MHz}$, the reflection coefficient $\mathrm{S} 11$ is as low as $-45 \mathrm{~dB}$, which is substantially lower than White et al.'s - $30 \mathrm{~dB}$; however, our measured $Q$ factor here was only 477 exciting a TE312 mode. It is important to note that there is no claimed preference to a specific mode; for example, Shawyer used a TE01 and TE11 mode in some of his experiments [16]. Our measurements cover up to four of them including TM and TE ones.

Thermocouples were used to monitor the thermal behavior of the amplifier, the fixed attenuator at the circulator, the RF cables, and the cavity near the emitter antenna. In this way, it was possible to ensure that RF power was fed into the cavity in resonant mode and to the fixed attenuator at anti-resonance. The amplifier temperature was logged to avoid overheating and degradation during measurements in vacuum.

\section{Measurement summary}

In order not to miss any thrust-effect, we performed a complete sweep from 1850 up to $2000 \mathrm{MHz}$ with a step size of $5 \mathrm{MHz}$, which included our tuned resonance frequency,
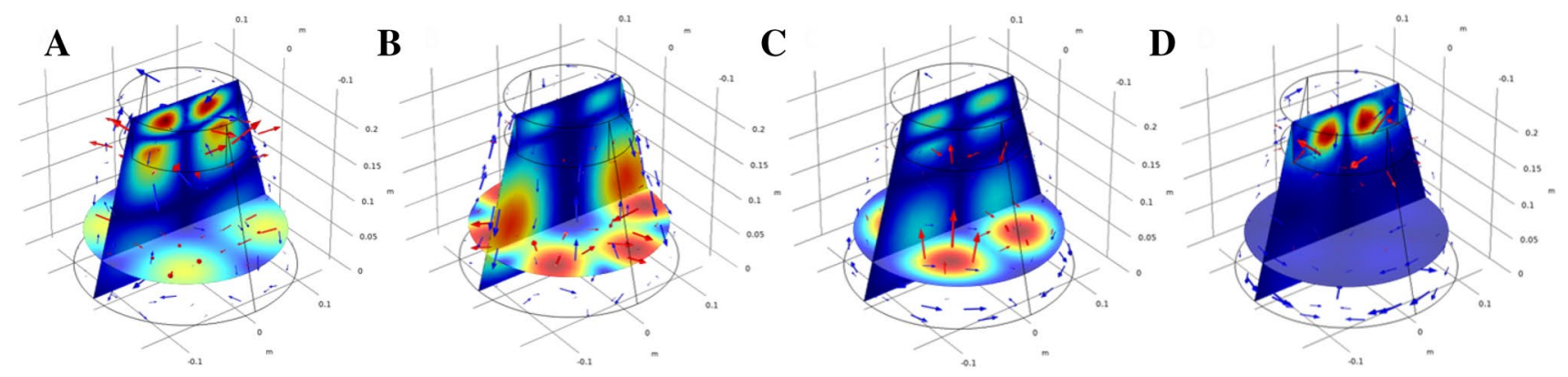

Fig. 10 Simulation of eigenfrequency modes corresponding to frequency peaks in Fig. 9. (A Hybrid mode, B TE312, C TM212, D hybrid mode). Electric field: red arrows; magnetic field: blue arrows 
other resonances, and data in between. More measurements with a smaller step size were taken close to the resonances. Figure 11 shows a summary of this effort, where each data point consists of an average of three single measurements at the respective frequency. The plot also shows the RF input power at the cavity for each frequency step. To obtain it, we did a separate calibration run by flipping the circulator, such that the output power from the amplifier was now not going into the cavity but directly into our power meter. This value was reduced by the circulator power measurement during the regular measurements, which then gave the real input power to the cavity. One can see that up to $11 \mathrm{~W}$ were going to the cavity at the same resonance frequencies as previously identified with the network analyzer in Fig. 9 and around $4 \mathrm{~W}$ outside the resonances. The real cavity power, as reported in Table 1, was estimated by subtracting the circulator power at resonance from the off-resonance value to account for the losses of the coaxial cable from the circulator to the three-stub tuner and connector to the antenna, based on datasheet values for the frequencies used. All thrust values were below our noise level of $10-30 \mathrm{nN}$.

From Eq. (1), we would have expected around $1 \mathrm{mN}$ using a design factor of 0.56 calculated from our used geometry, which is around 5 orders of magnitude above our measurements. Following the data from White et al. [3], we would have expected at least $10 \mu \mathrm{N}$, which is still three orders of magnitude above our data. However, such a value is similar to the false-positive thrust that we have seen due to thermally induced mechanical stress on the balance in Fig. 6b. Taking the $11 \mathrm{~W}$ for our photon force benchmark, this translates into $36 \mathrm{nN}$ as indicated by the shaded region in Fig. 11. Therefore, our measurements show that any anomalous thrust from an EMDrive must be on the order of or below the photon thrust limit. Considering that the amplifier alone required $60 \mathrm{~W}$ of electric power, the limit raises up $200 \mathrm{nN}$, which is an order of magnitude above our data.

Figure 12 shows the detail of a single frequency measurement close to our tuned resonance at $1914 \mathrm{MHz}$ together with the current that goes into the amplifier to see when power was on. We also see that the temperature on the amplifier raises by around 5 degrees, which can cause thermal drifts, shifts in the center of gravity and related issues as evaluated above. Only the correct mounting of the thruster package enabled us to get rid of these issues to reach the photon thrust sensitivity. Although some measurements were done with a frequency tracker targeting always minimum circulator power (= maximum cavity input power), we found that fixed frequency measurements were sufficient as the circular power monitor did not change throughout the measurement.

At last, Fig. 13 shows a thrust-frequency sweep from the 1850-2000 MHz range without taking individual profiles but by quickly scanning through all frequencies with very small steps. Again, only noise and no thrust signal are visible. We repeated the same measurement using a $300 \mathrm{~Hz}$ square wave to switch the amplifier on/off as suggested by Shawyer [9] without seeing any change. We also tested this pulse modulation with our false-positive signals and saw that the thrust decreased by about a factor of two consistent with the fact that in this case, the average power going to the cavity was reduced

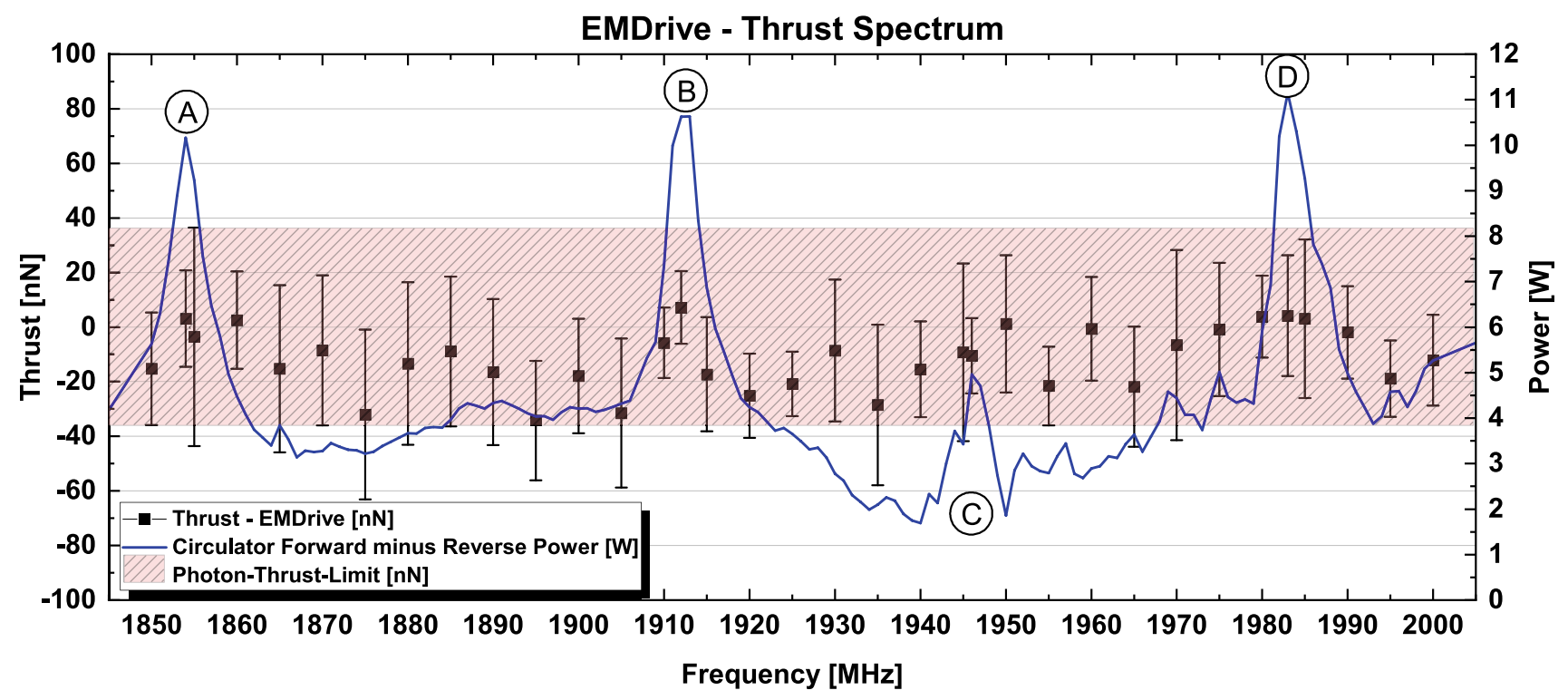

Fig. 11 Measurement of thrust spectrum in steps of $5 \mathrm{MHz}$ versus circulator forward power (peaks correspond to modes A-D); each measurement is an average of three single runs (photon-thrust limit equals to $11 \mathrm{~W}$ maximum input power) 
Fig. 12 Thrust measurement at fixed frequency $1914 \mathrm{MHz}$

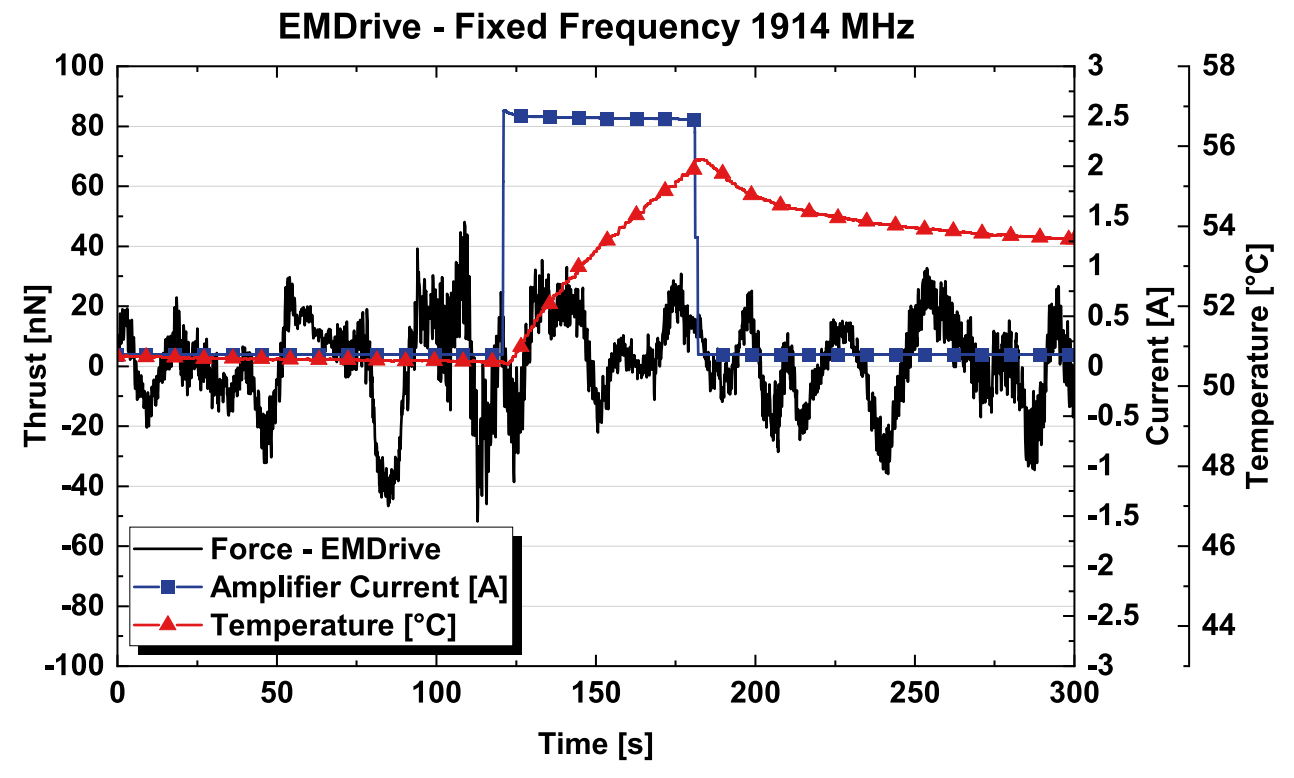

EMDrive - Fixed Frequency $1914 \mathrm{MHz}$

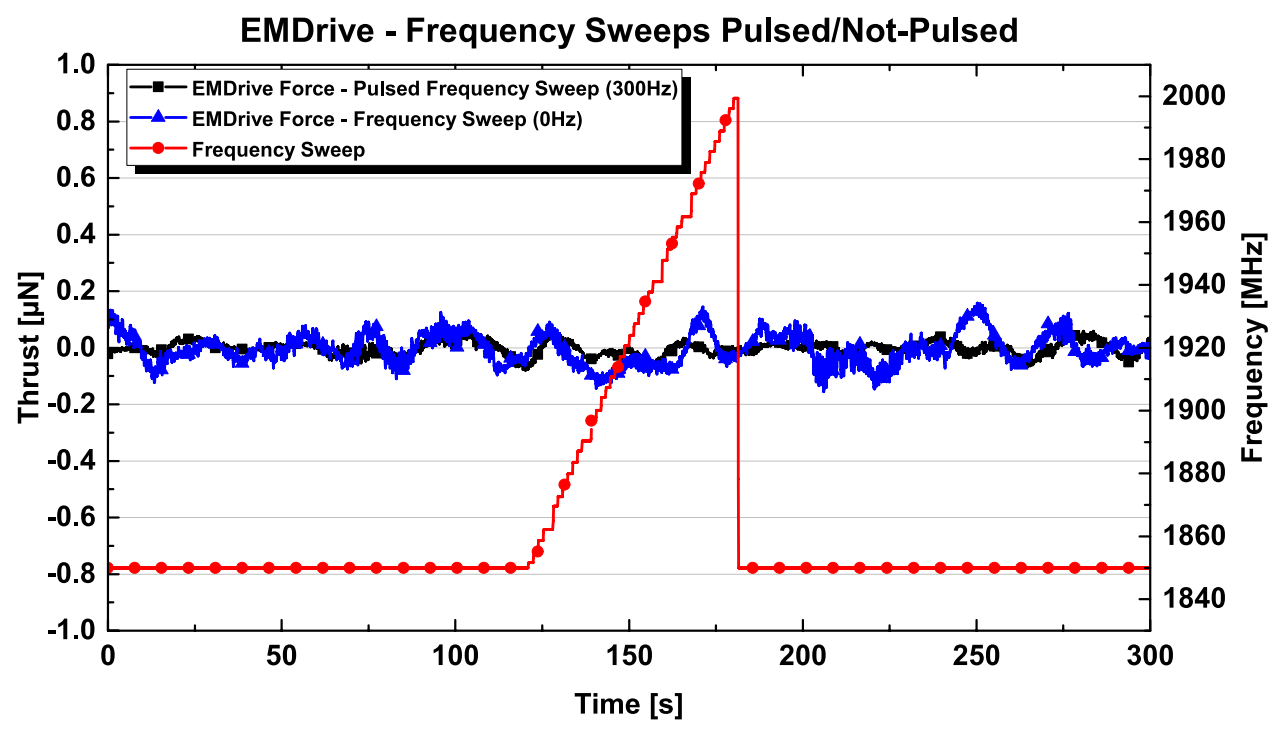

Fig. 13 Thrust-frequency sweep

by the same amount. Also, the claimed necessity of a pre-load was present in our setup as we did use torsion springs similar to the linear springs used in all setups by Shawyer [15-17].

\section{Conclusion and outlook}

We did a thorough assessment of previous measurements on the EMDrive and tested different balances and mounting options to identify the most critical measurement artefacts that can create false-positive thrust signatures, which can even pass consistency checks like being dependent on the orientation of the thruster on the balance. Finally, the development of an inverted counterbalanced double pendulum with bearing mounting and the assembly of a battery-powered EMDrive inside a sufficiently large vacuum chamber eliminated all mostly thermal-induced drifts as well as magnetic interaction. This allowed reaching a sensitivity equivalent to the force produced by the power going into the EMDrive and being radiated in a single direction like stateof-the-art propellant propulsion.

Our measurements span over a wide frequency range including four resonances and different modes as well as off-resonance regions. Our measurements limit any anomalous thrust to below the photon thrust limit, as summarized in Table 1. It should be noted that our Q factor was lower by an order of magnitude compared to others, which still rules out anomalous thrusts by at least two orders of magnitude according to Shawyer's Eq. (1). All data stayed within the limit of classical radiation pressure propulsion, which puts strong limits on all theories that were proposed in support of the EMDrive. 
Future work will concentrate on a superconducting cavity as suggested previously by Shawyer [9], which should boost the $\mathrm{Q}$ factor by many orders of magnitude to explore any anomalies also in this regime.

Acknowledgements We gratefully acknowledge the support for SpaceDrive by the German National Space Agency DLR (Deutsches Zentrum fuer Luft- und Raumfahrttechnik) by funding from the Federal Ministry of Economic Affairs and Energy (BMWi) by approval from German Parliament (50RS1704).

Funding Open Access funding enabled and organized by Projekt DEAL.

Open Access This article is licensed under a Creative Commons Attribution 4.0 International License, which permits use, sharing, adaptation, distribution and reproduction in any medium or format, as long as you give appropriate credit to the original author(s) and the source, provide a link to the Creative Commons licence, and indicate if changes were made. The images or other third party material in this article are included in the article's Creative Commons licence, unless indicated otherwise in a credit line to the material. If material is not included in the article's Creative Commons licence and your intended use is not permitted by statutory regulation or exceeds the permitted use, you will need to obtain permission directly from the copyright holder. To view a copy of this licence, visit http://creativecommons.org/licenses/by/4.0/.

\section{References}

1. Forward, R.L.: Feasibility of interstellar travel: A review. Acta Astronaut. 14, 243-252 (1986)

2. Shawyer, R.: Second generation EmDrive propulsion applied to SSTO launcher and interstellar probe. Acta Astronaut. 116, 166-174 (2015)

3. White, H., March, P., Lawrence, J., Vera, J., Sylvester, A., Brady, D. and Bailey, P.: Measurement of impulsive thrust from a closed radio-frequency cavity in vacuum. J. Propuls. Power 33(4), 830-841 (2017)

4. Tajmar, M., Kößling, M., Weikert, M., Monette, M.: The SpaceDrive project: Developing revolutionary propulsion at TU Dresden. Acta Astronaut. 153, 1-5 (2018)

5. Kößling, M., Monette, M., Weikert, M., Tajmar, M.: The SpaceDrive project: Thrust balance development and new measurements of the Mach-Effect and EMDrive Thrusters. Acta Astronaut. 161, 139-152 (2019)

6. Tajmar, M., Weikert, M., Monette, M.: The SpaceDrive Project: First results on EMDrive and mach-effect thrusters. In: Proceedings of the Space Propulsion Conference, SP2018_016 (2018)

7. Cullen, A.L.: Absolute power measurement at microwave frequencies. IEE Proc. 99(IV), 100-111 (1952)

8. Roger, S.: An EmDrive thruster for cubesats. In: Proceedings of the International Astronautical Congress, IAC, IAC-20,C4,6,9,x56845 (2020)

9. Shawyer, R.: EmDrive thrust load characteristics. In: Proceedings of the International Astronautical Congress, IAC, IAC-19-C4-10-14 (2019)

10. McCulloch, M.E.: Can the emdrive be explained by quantised inertia? Prog. Phys. 11(1), 78-80 (2015)

11. McCulloch, M.E.: Testing quantised inertia on emdrives with dielectrics. Europhys. Lett. 118(3), 34003 (2017)

12. Smolyaninov, I.: Optical analog of particle production in gravitational fields. Europhys. Lett. 128(5), 54002 (2020)
13. Grahn, P., Annila, A., Kolehmainen, E.: On the exhaust of electromagnetic drive. AIP Adv. 6(6), 065205 (2016)

14. Wu, C.W.: Comments on theoretical foundation of 'EM Drive.' Acta Astronaut. 144, 214-215 (2018)

15. Shawyer, R.J.: Technical report on the experimental microwave thruster. http://www.emdrive.com/FeasibilityStudytechnicalrepor tissue2.pdf (2002). Accessed 20 July 2021

16. Shawyer, R.J.: Technical report on the development of a microwave engine for satellite propulsion. http://www.emdrive.com/Demonstrat orTechnicalReportIssue2.pdf (2006). Accessed 20 July 2021

17. Shawyer, R.: Report on the design, development and test of a C-band flight thruster. http://www.emdrive.com/flighthrusterreportissue2. pdf (2017). Accessed 20 July 2021

18. Shawyer, R.: Notes on demonstrator engine dynamic tests. http:// www.emdrive.com/testnotes.pdf (2015). Accessed 20 July 2021

19. Yang, J., Yu-Quan, W., Yan-Jie, M., Peng-Fei, L., Le, Y., Yang, W. and Guo-Qiang, H.: Prediction and experimental measurement of the electromagnetic thrust generated by a microwave thruster system. Chin. Phys. B 22(5), 050301 (2013)

20. Yang, J., Liu, X., Wang, M., Tang, Y., Luo, L., Jin, Y., Ning, Z.: Thrust measurement of an independent microwave thruster propulsion device with three-wire torsion pendulum thrust measurement system. J. Propuls. Technol. 37(2), 362-371 (2016)

21. Fetta, G. P.: Numerical and experimental results for a novel propulsion technology requiring no on-board propellant. In: 50th AIAA/ ASME/SAE/ASEE Joint Propulsion Conference, AIAA 2014-3853 (2014)

22. Brady, D., White, H., March, P., Lawrence, J., Davies, F.: Anomalous thrust production from an RF test device measured on a lowthrust torsion pendulum. In: 50th AIAA/ASME/SAE/ASEE Joint Propulsion Conference, AIAA 2014-4029 (2014)

23. McDonald, M.S., Nurnberger, M.W., Williams, L.T.: Preparations for thrust measurement and error discussion of the impulse resonant microwave cavity. J. Br. Interplanet. Soc. 70(10-11), 415-424 (2017)

24. Sokoloff, J., Pascal, O., Pigaglio, O., Raveu, N., Peyre, H.: Proposal of a handy setup for discriminating parasitic effects for the measurement of impulsive thrust from a microwave cavity. Prog. Electromagn. Res. C 98, 269-281 (2020)

25. Peyre, H., Sokoloff, J., Pascal, O., Pigaglio, O., Raveu, N.: Straightforward EMDrive setup using a microwave contactless transition: first results on frustum cavities. Prog. Electromagn. Res. M 95, 45-52 (2020)

26. Peyre, H., Sokoloff, J., Pascal, O., Pigaglio, O., Raveu, N.: Straightforward Emdrive setup with nasa-like cavities. Prog. Electromagn. Res. M 101, 1-8 (2021)

27. Tajmar, M., Fiedler, G.: Direct thrust measurements of an EMDrive and evaluation of possible side-effects. In: 51st AIAA/SAE/ASEE Joint Propulsion Conference, AIAA 2015-4083 (2015)

28. Neunzig, O., Weikert, M., Tajmar, M.: Characterization of a rotational thrust balance for propellantless propulsion concepts utilizing magnetic. In: 36th International Electric Propulsion Conference, IEPC-2019-290 (2019)

29. Taylor, T.S.: Propulsive forces using high-Q asymmetric high energy laser resonators. J. Br. Interplanet. Soc. 70(7), 238-243 (2017)

30. Neunzig, O., Weikert, M., Tajmar, M.: Thrust measurements and evaluation of asymmetric infrared laser resonators for space propulsion. In: Proceedings of the 7th Space Propulsion Conference, SP2020_0104 (2021)

31. Kößling, M., Weikert, M., Tajmar, M.: Experimental evaluation of the VEM drive," VEM-GWT-TN-1001. https://nbn-resolving.org/ urn:nbn:de:bsz:14-qucosa2-707098 (2020). Accessed 20 July 2021

32. Tajmar, M., Schreiber, T.: Put strong limits on all proposed theories so far assessing electrostatic propulsion: Does a charged high-voltage capacitor produce thrust? J. Electrostat. 107, 103477 (2020) 
33. Polk, J. E., Haag, T., King, S., Walker, M., Blakely, J. and Ziemer, J.: Recommended practices in thrust measurements. In: 33rd International Electric Propulsion, IEPC-2013-440 (2013)

34. Walker, B.: Determining resonator $\mathrm{Q}$ factor from return loss measurement alone. https://coppermountaintech.com/determiningresonator-q-factor-from-return-loss-measurement-alone/. (2021). Accessed 30 Apr 2021
Publisher's Note Springer Nature remains neutral with regard to jurisdictional claims in published maps and institutional affiliations. 